\title{
ASTROPHYSICAL PARAMETERS OF LS 2883 AND IMPLICATIONS FOR THE PSR B1259-63 GAMMA-RAY BINARY*
}

\author{
Ignacio Negueruela ${ }^{1}$, Marc Ribó ${ }^{2}$, Artemio Herrero ${ }^{3,4}$, Javier Lorenzo $^{1}$, \\ Dmitry Khangulyan ${ }^{5}$, and Felix A. Aharonian ${ }^{6,7}$ \\ ${ }^{1}$ Departamento de Física, Ingeniería de Sistemas y Teoría de la Señal, Universidad de Alicante, Apdo. 99, E-03080 Alicante, Spain; \\ ignacio.negueruela@ua.es, javihd64315@gmail.com \\ ${ }^{2}$ Departament d'Astronomia i Meteorologia, Institut de Ciènces del Cosmos (ICC), Universitat de Barcelona (IEEC-UB), \\ Martí i Franquès 1, E-08028 Barcelona, Spain; mribo@am.ub.es \\ ${ }^{3}$ Instituto de Astrofísica de Canarias, E-38200 La Laguna, Spain; ahd@iac.es \\ ${ }^{4}$ Departamento de Astrofísica, Universidad de La Laguna, Avda. Astrofísico Francisco Sánchez, s/n, E-38205 La Laguna, Spain \\ ${ }^{5}$ Institute of Space and Astronautical Science/JAXA, 3-1-1 Yoshinodai, Chuo-ku, Sagamihara, Kanagawa 252-5210, Japan; khangul@ astro.isas.jaxa.jp \\ ${ }^{6}$ Dublin Institute for Advanced Studies, 31 Fitzwilliam Place, Dublin 2, Ireland; felix.aharonian@ dias.ie \\ ${ }^{7}$ Max-Planck-Institut für Kernphysik, Saupfercheckweg 1, D-69117 Heidelberg, Germany \\ Received 2010 November 8; accepted 2011 March 22; published 2011 April 8
}

\begin{abstract}
Only a few binary systems with compact objects display $\mathrm{TeV}$ emission. The physical properties of the companion stars represent basic input for understanding the physical mechanisms behind the particle acceleration, emission, and absorption processes in these so-called gamma-ray binaries. Here we present high-resolution and high signalto-noise optical spectra of LS 2883 , the Be star forming a gamma-ray binary with the young non-accreting pulsar PSR B1259-63, showing it to rotate faster and be significantly earlier and more luminous than previously thought. Analysis of the interstellar lines suggests that the system is located at the same distance as (and thus is likely a member of) Cen OB1. Taking the distance to the association, $d=2.3 \mathrm{kpc}$, and a color excess of $E(B-V)=0.85$ for LS 2883 results in $M_{V} \approx-4.4$. Because of fast rotation, LS 2883 is oblate $\left(R_{\text {eq }} \simeq 9.7 R_{\odot}\right.$ and $\left.R_{\text {pole }} \simeq 8.1 R_{\odot}\right)$ and presents a temperature gradient $\left(T_{\text {eq }} \approx 27,500 \mathrm{~K}, \log g_{\text {eq }}=3.7 ; T_{\text {pole }} \approx 34,000 \mathrm{~K}, \log g_{\text {pole }}=4.1\right)$. If the star did not rotate, it would have parameters corresponding to a late O-type star. We estimate its luminosity at $\log \left(L_{*} / L_{\odot}\right) \simeq 4.79$ and its mass at $M_{*} \approx 30 M_{\odot}$. The mass function then implies an inclination of the binary system $i_{\text {orb }} \approx 23^{\circ}$, slightly smaller than previous estimates. We discuss the implications of these new astrophysical parameters of LS 2883 for the production of high-energy and very high-energy gamma rays in the PSR B1259-63/ LS 2883 gamma-ray binary system. In particular, the stellar properties are very important for prediction of the line-like bulk Comptonization component from the unshocked ultrarelativistic pulsar wind.
\end{abstract}

Key words: binaries: close - gamma rays: stars - pulsars: individual (PSR B1259-63) - stars: emission-line, Be stars: individual (LS 2883) - X-rays: binaries

Online-only material: color figures

\section{INTRODUCTION}

Three binary systems containing a massive star and a compact object that clearly display $\mathrm{TeV}$ emission are currently known: LS 5039, LS I +61 303 and PSR B1259-63 (see, e.g., Ribó 2008). In PSR B1259-63 the compact object is a neutron star, first discovered as a $47.7 \mathrm{~ms}$ radio pulsar, in a very wide and eccentric orbit $\left(e=0.87, P_{\text {orb }}=1236.9\right.$ days $)$ around the Be star LS $2883^{8}$ (Johnston et al. 1992, 1994). A doublepeaked non-thermal and unpulsed radio outburst takes place around each periastron passage (Johnston et al. 2005). This radio emission has recently been resolved at AU scales by Moldón et al. (2011), who place it outside the binary system. Doublepeaked outbursts have also been detected in soft and hard X-rays, and at TeV energies by HESS (Uchiyama et al. 2009; Grove et al. 1995; Aharonian et al. 2005, 2009). Upper limits were obtained

\footnotetext{
* Partially based on observations collected at the European Southern Observatory, Paranal, Chile (ESO 282.D-5081), and the South African Astronomical Observatory.

8 Star 2883 in the catalog of Luminous Stars in the Southern Milky Way (Stephenson \& Sanduleak 1971) is also known as CPD $-63^{\circ} 2495$. The use of SS 2883 should be avoided, as this acronym refers to the catalog of emission-line stars by Stephenson \& Sanduleak (1977), which contains only 455 stars.
}

at $\mathrm{GeV}$ energies by EGRET around the 1994 periastron passage (Tavani et al. 1996).

In the currently preferred scenario, the cold ultrarelativistic wind of the pulsar interacts with the massive star wind within the binary system. Relativistic electrons are accelerated in a shock region where the pressures of both winds balance. These electrons produce synchrotron radiation and upscatter UV-optical photons from the companion star via inverse Compton (IC), giving rise to broadband emission up to $\mathrm{TeV}$ gamma rays (e.g., Kirk et al. 1999; Dubus 2006; Khangulyan et al. 2007; Aharonian et al. 2009). GeV and $\mathrm{TeV}$ emission can also be produced from IC scattering of stellar photons by electrons in the unshocked pulsar wind, depending on its Lorentz factor. Radio emission outside the binary system is produced by long-lived particles traveling away in a kind of cometary tail.

In these models, the companion star is the source of seed photons for IC scattering, expected to be the dominant radiation mechanism. Fundamental parameters, like the luminosity or the effective temperature, have to be known accurately to properly model, and thus be able to understand, how this gamma-ray binary produces the observed multiwavelength properties (see Khangulyan et al. 2007). LS 2883 was observed in the optical by Johnston et al. (1994), who identified emission lines typical of a Be star. Based on the presence of strong He I emission, 


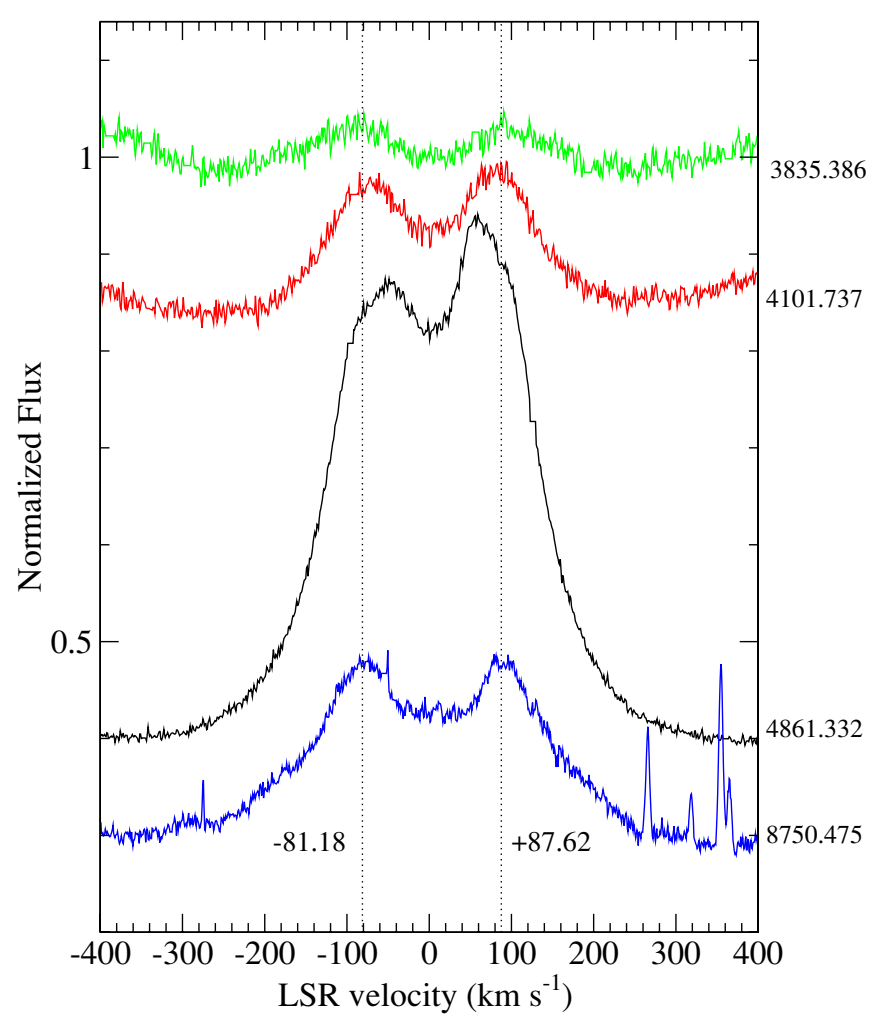

Figure 1. Representative emission lines in the VLT/UVES spectrum of LS 2883, in velocity space. Velocities are measured with respect to the local standard of rest (LSR) assuming solar motion $+16.6 \mathrm{~km} \mathrm{~s}^{-1}$ toward Galactic coordinates $\ell=53^{\circ}, b=+25^{\circ}$. From top to bottom and vertically shifted for clarity, we show $\mathrm{H} \eta$ (green), $\mathrm{H} \delta$ (red), $\mathrm{H} \beta$ (black), and $\mathrm{Pa} 11$ (blue). Their central wavelengths are quoted in $\AA$ to the right of the figure. The dotted lines mark the position of the emission peaks in $\mathrm{Pa} 11$, with velocities in $\mathrm{km} \mathrm{s}^{-1}$ annotated.

(A color version of this figure is available in the online journal.)

they concluded that it was earlier than B3. Arguing that the star was likely located in the Sagittarius Arm, Johnston et al. (1994) assumed a distance of $1.5 \mathrm{kpc}$ and a spectral type B2e.

Here we report on new astrophysical parameters of LS 2883, a new distance estimate and a new inclination of the binary orbit. These results will allow for better modeling of the multiwavelength data acquired close to the periastron passage of PSR B1259-63 in 2010 mid-December, the first covered by the Fermi and AGILE gamma-ray missions.

\section{OBSERVATIONS AND SPECTRUM DESCRIPTION}

Observations of LS 2883 were obtained on 2009 August 28 using the Ultraviolet and Visual Echelle Spectrograph (UVES; Dekker et al. 2000) mounted on the $8.2 \mathrm{~m}$ Very Large Telescope (VLT) UT2 Kueyen at Cerro Paranal, Chile. UVES was used in dichroic 2 mode with cross-disperser CD\#2 in the blue arm, giving coverage of the 373-499 $\mathrm{nm}$ range, and CD\#4 in the red arm, allowing coverage of 565-946 nm with a small gap around $760 \mathrm{~nm}$. We used Image Slicer 2 and a 0.'5 slit, giving a resolving power $R \sim 80,000$.

In addition, intermediate-resolution spectra of LS 2883 and three nearby blue stars were obtained on 2006 May 8, using the unit spectrograph on the $1.9 \mathrm{~m}$ telescope at the South African Astronomical Observatory (SAAO) in Sutherland ${ }^{9}$ with grating $6\left(600\right.$ lines $\left.\mathrm{mm}^{-1}\right)$. This configuration covers $380-560 \mathrm{~nm}$ with $R \approx 2000$ (measured on arc frames).

\footnotetext{
9 http://www.saao.ac.za/facilities/instrumentation/gratingspec/
}

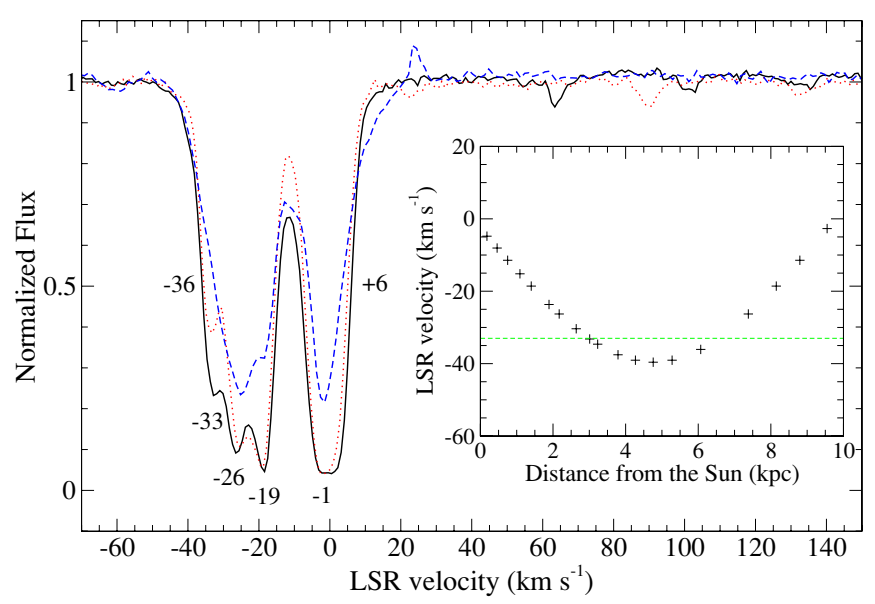

Figure 2. Interstellar lines in the VLT/UVES spectrum of LS 2883. The main panel shows the components of three of the strongest interstellar lines: $\mathrm{NaI}$ $5890 \AA$ A (solid black), Na I $5896 \AA$ A (dotted red), and Ca II $3934 \AA$ (dashed blue) in velocity space. Peaks in the absorption and the extreme edges are annotated. The inset shows the Galactic rotation curve (black crosses) along this line of sight $(\ell=304.2, b=-1$. 0 ) according to Brand \& Blitz (1993), using a circular rotation velocity at the position of the $\operatorname{Sun}\left(d_{\mathrm{GC}}=8.5 \mathrm{kpc}\right)$ of $220 \mathrm{~km} \mathrm{~s}^{-1}$. The green dashed line indicates the velocity of the most negative peak measured toward LS 2883.

(A color version of this figure is available in the online journal.)

The spectrum of LS 2883 is typical of a Be star of early type with a well-developed disk (cf. Johnston et al. 1994). Balmer lines have strong double-peaked emission components, with the red peak clearly stronger than the blue one (the two peaks are blended in $\mathrm{H} \alpha$ ). Representative emission lines are displayed in Figure 1. All Balmer lines higher than $\mathrm{H} \gamma$ clearly show the photospheric absorption wings, as the emission feature is definitely narrower than the absorption line, allowing the determination of effective gravity. Paschen lines between $\mathrm{Pa} 11$ and $\mathrm{Pa} 23$ do not show any sign of the photospheric component. Other emission lines typical of early-B stars, such as O I $8448 \AA$ or He I 5875, 6678, $7065 \AA$ appear as doublepeaked features. The O I $7775 \AA$ triplet is blended into a strong single feature. There are many weak emission lines, mostly from Fe II transitions, but also including Si II 4128 and 6347 A. The SAAO spectrum also shows strong emission lines from Fe II 5019, 5169, and $5316 \AA$.

The equivalent width of $\mathrm{H} \alpha, \mathrm{EW}(\mathrm{H} \alpha)$, is $-54 \pm 2 \AA$, significantly higher than measured by Johnston et al. (1994), $-40 \AA$, though the overall shape is very similar. The $\operatorname{EW}(\mathrm{H} \beta)$ is $-4.5 \pm 0.4$ in the 2006 SAAO spectrum and $-4.6 \pm 0.2$ in the 2009 VLT/UVES spectrum, showing no significant variability. As seen in Figure 1, all the upper Paschen and Balmer features have similar peak separation, with values $170 \pm 10 \mathrm{~km} \mathrm{~s}^{-1}$.

\section{ASTROPHYSICAL PARAMETERS}

\subsection{Distance and Color Excess Estimates}

Since model fitting can only give values for $T_{\text {eff }}$ and $\log g$, we need an accurate distance to LS 2883 to derive $M_{*}$ and $L_{*}$. We use interstellar atomic lines in the spectrum of LS 2883 to study the radial velocity distribution of material along this line of sight ( $\ell=304.2, b=-1.0)$. Figure 2 displays three strong interstellar lines in the spectrum of LS 2883, the two components of the $\mathrm{Na}$ I doublet and one of the members of the $\mathrm{Ca}$ II doublet. All three lines (and other interstellar lines, like CN $3875 \AA$ ) show the same morphology, with two clearly separated components. 
The first component, with low positive velocity, is likely due to nearby clouds associated with the Southern Coalsack, which shows local standard of rest (LSR) velocities in the range -10 to $+8 \mathrm{~km} \mathrm{~s}^{-1}$ in CO maps (Nyman et al. 1989). The second feature is a combination of several weaker components with negative velocities (between $-14 \mathrm{~km} \mathrm{~s}^{-1}$ and $-36 \mathrm{~km} \mathrm{~s}^{-1}$ ), values produced by clouds in the Sagittarius Arm. Very similar components are observed in the interstellar lines of HD 112272 $(\ell=303.5, b=-1.5)$, a B0.5 Ia supergiant believed to be a member of Cen OB1 (Hunter et al. 2006).

In this direction, the H I shell GSH $305+01-24$, with dynamical $d=2.2 \pm 0.6 \mathrm{kpc}$, has an average velocity $-24 \mathrm{~km} \mathrm{~s}^{-1}$ and is believed to be associated to Cen OB1 (McClure-Griffiths et al. 2002). LS 2883 shows interstellar components more negative than this value, suggesting that it is beyond this shell. In fact, the Galactic rotation curve would place LS 2883 at $\gtrsim 2.5 \mathrm{kpc}$ (see the inset of Figure 2). More distant stars along this sight line show narrow components at $\approx-50 \mathrm{~km} \mathrm{~s}^{-1}$, believed to arise from clouds in the Norma-Centaurus Arm, at $d \gtrsim 4 \mathrm{kpc}$ (Kaper et al. 2006, and references therein). The lack of this component in LS 2883 sets an upper limit to its distance and firmly places it near Cen OB1.

Photometric measurements of LS 2883 in the literature (Klare \& Neckel 1977; Schild et al. 1983; Westerlund \& Garnier 1989; Drilling 1991) suggest some variability, at the level of a few hundredths of a magnitude, typical of Be stars. Taking $(B-V)=0.73$ as representative, the intrinsic color corresponding to the model fit (see below), $(B-V)_{0}=-0.28$, implies $E(B-V)=1.01$. Not all this reddening is interstellar, as the disks of Be stars give rise to circumstellar reddening due to stronger emission at longer wavelengths (e.g., Dachs et al. $1988)$. The correlation between $\mathrm{EW}(\mathrm{H} \alpha)$ and $E^{\mathrm{cs}}(B-V)$ from Dachs et al. (1988), valid solely for isolated Be stars, predicts $E^{\mathrm{cs}}(B-V)=0.11$, higher than for most Be stars. Higher values are observed in $\mathrm{Be} / \mathrm{X}$-ray binaries, but values $\gtrsim 0.3 \mathrm{mag}$ are generally associated with transient events (e.g., Reig et al. 2007). Therefore, it seems sensible to assume LS 2883 to have interstellar $E(B-V)=0.8-0.9 \mathrm{mag}$.

We have also estimated the color excess using four interstellar diffuse bands (DIBs) with EW $>100 \mathrm{m \AA}$ in the spectrum of LS 2883: $\lambda 5780, \lambda 5797$ (Herbig 1993), $\lambda 6202$, and $\lambda 6614$ (Rawlings et al. 2003). $\lambda 4430$ is difficult to measure. Though there is some dispersion, all support $E(B-V)$ between $0.8-$ $0.9 \mathrm{mag}$. We shall thus assume $E(B-V)=0.85$. Members of Cen OB1 near LS 2883 have $E(B-V)$ between 0.7 and 1.1 (Humphreys 1978).

The extinction law in this direction does not differ significantly from the average $R=3.1$ law (Winkler 1997). We observed two luminous stars close to LS 2883 from SAAO. For LS 2888 ( $7^{\prime}$ away), we derive a spectral type B0.2 III. Using photometry from the literature (Klare \& Neckel 1977; Schild et al. 1983) and standard calibrations for intrinsic colors (Fitzgerald 1970) and magnitudes (Turner 1980), we estimate $E(B-V)=0.83$ and $d=2.9 \pm 0.5 \mathrm{kpc}$. For LS 2882, only $30^{\prime \prime}$ away from LS 2883, we derive a spectral type B1 IV. Unfortunately, there is no accurate photometry published for this star. Its 2 MASS $\left(J-K_{\mathrm{S}}\right)$ color, however, indicates that it is reddened by an amount very similar to LS 2888. The spectral types and magnitudes are fully compatible with the three stars being at similar distances.

Therefore, direct measurements and indirect evidence are consistent with the idea that LS 2883 has interstellar $E(B-V)=$ $0.85 \pm 0.05$ and is a member of Cen OB1. The distance to this

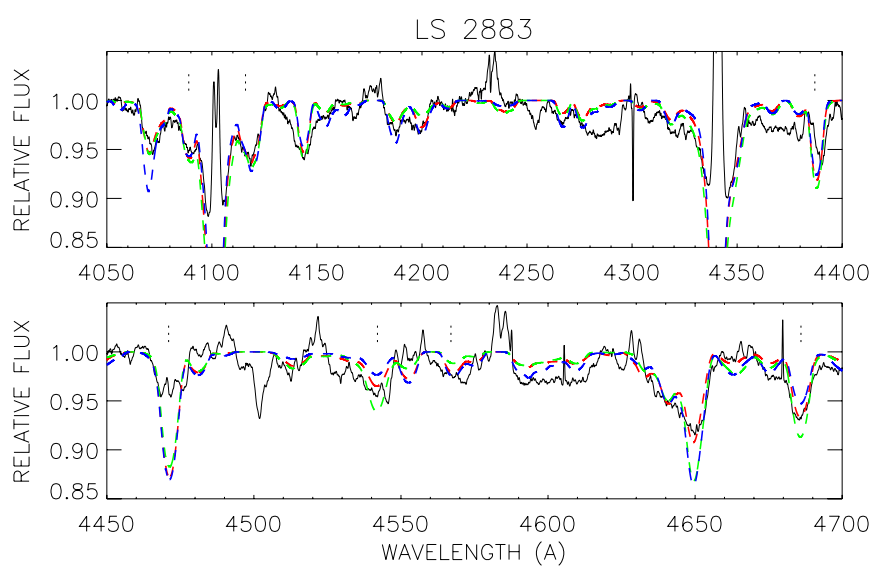

Figure 3. Rectified VLT/UVES blue spectrum of LS 2883 (solid black) and its best model fit (dashed red). To illustrate the range of acceptable values, in the top panel we also show models calculated with $T_{\text {eff }}=32,000 \mathrm{~K}$ and $\log g=3.60$ (blue dashes) and $\log g=4.10$ (green dashes). In the lower panel, we show models with $\log g=3.80$ and $T_{\text {eff }}=31,000 \mathrm{~K}$ (blue dashes) and $T_{\text {eff }}=34,000 \mathrm{~K}$ (green dashes). Features used for the analysis are marked with dashed lines.

(A color version of this figure is available in the online journal.)

association has been studied by different authors, with most recent values converging toward $d=2.3 \mathrm{kpc}$, though values up to $d=2.5 \mathrm{kpc}$ are reported (Kaltcheva \& Georgiev 1994; Humphreys 1978, and references therein). We will assume that LS 2883 is located at the distance to the association, even though values as high as $2.8 \mathrm{kpc}$ seem compatible with the data available. Values lower than $2.0 \mathrm{kpc}$ seem to be excluded by the kinematic data contained in the interstellar lines to LS 2883.

\subsection{Spectrum Modeling}

Stellar parameters have been calculated using FASTWIND (Puls et al. 2005), a spherical non-LTE model atmosphere code with mass loss, developed to model detailed spectral line profiles of hot and luminous stars. The best-fit model is shown in Figure 3.

We determined the stellar rotational velocity using the Fourier transform method. We first degraded the spectrum to $R=$ 10,000 for a better signal-to-noise ratio. Because of the large broadening, many lines are blended. We took unblended line wings and mirrored them (thus producing a symmetric line) before calculating the Fourier transform. This procedure gives results consistent with measurements for the blended lines. Our values, which rest on the metallic lines O II $4254 \AA$ and $\mathrm{C}_{\text {II }} 4267 \AA$, are $v_{\text {rot }} \sin i=235 \pm 15 \mathrm{~km} \mathrm{~s}^{-1}$ (projected rotational velocity; to be refined further below) and $\zeta=$ $115 \pm 50 \mathrm{~km} \mathrm{~s}^{-1}$ (so-called macroturbulence). Errors simply represent the dispersion of individual values.

Stellar parameters were obtained by visual fitting to the Balmer, He I/He II and Si III/Si IV lines (see, e.g., Repolust et al. 2004; Simón-Díaz 2010). The fit is not as accurate as in these works, since the heavy veiling by emission lines makes the continuum definition a difficult task and some diagnostic lines either have emission components (such as He I $4471 \AA$ ) or are affected by nearby emission lines (as, for example, Si III $4552 \AA$ ). The best fit is obtained for $T_{\text {eff }}=32,000_{-1000}^{+2000} \mathrm{~K}$ and $\log g=3.8_{-0.2}^{+0.3}$, where the uncertainties have been obtained following the procedures discussed in Section 6 of Repolust et al. (2004). A normal He abundance $(N(\mathrm{He}) /(N(\mathrm{H})+N(\mathrm{He}))=$ $0.09)$ is consistent with the observed spectrum, although a 
Table 1

Fitted and Derived Apparent Stellar Parameters for LS 2883

\begin{tabular}{lc}
\hline \hline Parameter & Value \\
\hline$T_{\text {eff }}(\mathrm{K})$ & $32000_{-1000}^{+2000}$ \\
$\log g$ & $3.80_{-0.20}^{+0.30}$ \\
$R_{*}{ }^{\mathrm{a}}\left(R_{\odot}\right)$ & $9.0_{-1.5}^{+1.8}$ \\
$\log \left(L_{*} / L_{\odot}\right)^{\mathrm{a}}$ & $4.88_{-0.17}^{+0.19}$ \\
$M_{*}{ }^{\mathrm{a}}\left(M_{\odot}\right)$ & $21.3_{-9.0}^{+22.0}$ \\
$M_{*}{ }^{\mathrm{a}, \mathrm{b}}\left(M_{\odot}\right)$ & $26.6_{-10.5}^{+23.5}$ \\
\hline
\end{tabular}

Notes.

${ }^{\text {a Assuming }} d=2.3 \pm 0.4 \mathrm{kpc}$ and $E(B-V)=0.85 \pm 0.05$.

b Deprojecting the rotational velocity with $i=35^{\circ}$, after applying the correction of Fremat et al. (2005).

slightly increased $\mathrm{He}$ abundance is possible. The lines typically used to estimate the mass-loss rate are strongly contaminated by emission components, preventing us from obtaining an accurate value. We have adopted the mass-loss rate expected for a spherical star of the luminosity derived below according to the recipes of Vink et al. (2000), resulting in a thin wind with no effect on the emergent profiles.

Using $d=2.3 \pm 0.4 \mathrm{kpc}, E(B-V)=0.85 \pm 0.05$, and $R=3.1 \pm 0.1$, we derive $M_{V}=-4.4 \pm 0.4$. From the modelfit spectrum and the absolute magnitude, we derive the values for $R_{*}, L_{*}$, and $M_{*}$ listed in Table 1 . The fitted parameters correspond to an $09.5 \mathrm{~V}$ star according to the observational scale of Martins et al. (2005), in good agreement with the morphological spectral classification that we derive from our spectra.

However, for fast rotators, there are strong differences in effective gravity between the polar and equatorial regions, resulting in important temperature gradients and oblateness (Townsend et al. 2004, and therein). The binary mass function $f\left(M_{\mathrm{NS}}\right)=1.53 M_{\odot}$ (Johnston et al. 1994) implies an orbital inclination angle of $i_{\text {orb }}=24.7_{-5.4}^{+5.9}$ for a standard neutron star mass of $1.4 M_{\odot}$. Melatos et al. (1995) find a tilt of $10^{\circ}$ between the orbital plane and circumstellar disk, expected to lie on the stellar equatorial plane. Therefore, we are seeing LS 2883 under a low $\left(\sim 35^{\circ}\right)$ inclination and the parameters obtained must mainly reflect the characteristics of the hotter polar region.

For a centrally condensed star, the critical rotational angular velocity (gravity and centrifugal forces are equal at the equator) is given by

$$
\Omega_{\text {crit }}=\sqrt{\frac{8 G M_{*}}{27 R_{\text {pole }}^{3}}} .
$$

A star rotating at $\Omega_{\text {crit }}$ will have $R_{\text {eq }}=1.5 R_{\text {pole }}($ Cranmer $\&$ Owocki 1995). In the case of LS 2883, with a deprojected $v_{\text {rot }}=$ $410 \mathrm{~km} \mathrm{~s}^{-1}$, a rough estimation suggests that $R_{\mathrm{eq}}>1.1 R_{\text {pole }}$ and $\omega \equiv \Omega_{*} / \Omega_{\text {crit }} \gtrsim 0.85$, meaning that two-dimensional effects will be important.

For a start, in fast rotators, both the Full Width at Half Maximum (FWHM) of lines and the Fourier method underestimate $v_{\text {rot }} \sin i$ (Townsend et al. 2004; Fremat et al. 2005). We therefore correct $v_{\text {rot }} \sin i$ using Figure 8 of Fremat et al. (2005), finding $v_{\text {rot }}^{\prime} \sin i=260 \pm 15 \mathrm{~km} \mathrm{~s}^{-1}$. The deprojected velocity is then $v_{\text {rot }}^{\prime} \approx 450 \mathrm{~km} \mathrm{~s}^{-1}$, even closer to the critical value.

Though a complete two-dimensional treatment of the stellar surface is beyond our scope, we can perform a quick evaluation of the effects of fast rotation. From the $T_{\text {eff }}$ and $\log g$ derived from our analysis (apparent values), we estimate the $T_{\text {eff }}, \log g$, and $M_{V}^{0}$ of a non-rotating star that would give the same apparent
Table 2

Estimated Actual Stellar Parameters for LS 2883

\begin{tabular}{lc|lc}
\hline \hline \multicolumn{2}{c|}{ Non-rotating } & \multicolumn{2}{c}{ Rotating } \\
\hline$T_{\text {eff }}^{0}$ & $33500 \mathrm{~K}$ & $i$ & $33^{\circ}$ \\
$\log g^{0}$ & 4.0 & $\omega$ & 0.88 \\
$M_{V}^{0}$ & -4.47 & $T_{\text {eff }}(\mathrm{eq})$ & $27500 \mathrm{~K}$ \\
$R_{*}^{0}$ & $9.2 R_{\odot}$ & $T_{\text {eff }}($ pole $)$ & $34000 \mathrm{~K}$ \\
$\log \left(L_{*}^{0} / L_{\odot}\right)$ & 4.98 & $\log g($ eq $)$ & 3.7 \\
$M_{*}^{0}$ & $31 M_{\odot}$ & $\log g($ pole $)$ & 4.1 \\
& & $R_{\text {eq }}$ & $9.7 R_{\odot}$ \\
& & $R_{\text {pole }}$ & $8.1 R_{\odot}$ \\
& & $\log \left(L_{*} / L_{\odot}\right)$ & 4.79 \\
\hline
\end{tabular}

parameters when rotating at the same $v_{\text {rot }}$ as LS 2883. For this, we use an iterative method based on the calculations of Fremat et al. (2005) and the tables of Collins et al. (1991). With these new values, we calculate other stellar parameters and iterate again to take into account the change in the inclination due to the change in $M_{*}$. The results of this procedure are the stellar parameters that LS 2883 would have if it did not rotate (Table 2, left panel) and its most likely actual parameters, taking into account fast rotation (Table 2, right panel).

LS 2883 presents important differences in $T_{\text {eff }}$ and $\log g$ between the polar and equatorial regions, rendering the apparent parameters little more than an approximate guess. The hypothetical non-rotating LS 2883 would have parameters roughly corresponding to an $\mathrm{O} 8 \mathrm{~V}$ star. Its observed spectrum is a consequence of fast rotation. The mass derived, $31 M_{\odot}$, is somewhat high for these parameters, but is subject to large uncertainties, as it depends strongly on the corrections for fast rotation and the distance assumed.

\section{DISCUSSION}

Our spectroscopic observations and atmosphere model fitting have provided new physical parameters for LS 2883, the massive star forming a gamma-ray binary with the young non-accreting pulsar PSR B1259-63. The higher temperature and luminosity of the optical star presented here, $T_{\mathrm{eq}} \approx$ $27,500 \mathrm{~K}, T_{\text {pole }} \approx 34,000 \mathrm{~K}, L_{*}=2.3 \times 10^{38} \mathrm{erg} \mathrm{s}^{-1}$, as compared to previous estimates, $T_{\text {eff }}=23,000-27,000 \mathrm{~K}$, $L_{*}=(0.3-2.2) \times 10^{38} \mathrm{erg} \mathrm{s}^{-1}$ (see Khangulyan et al. 2007), imply a significant revision of the parameters and conditions for the production of non-thermal radiation in this binary system, especially in the gamma-ray band. High $(\mathrm{GeV})$ and very high $(\mathrm{TeV})$ energy gamma rays from this system can be produced in two distinct regions: (1) in the unshocked pulsar wind, i.e., a cold ultrarelativistic outflow expanding outward from the pulsar with Lorentz factor $\Gamma \leqslant 10^{6}$; and (2) in the region of the terminated pulsar wind. In both regions, the dominant gammaradiation mechanism is IC scattering of relativistic electrons. While the very high energy (VHE) emission detected by HESS is most likely linked to the multi-TeV electrons accelerated after termination of the wind, $\mathrm{GeV}$ gamma-ray emission can be effectively produced also by the unshocked pulsar wind. The higher luminosity of the optical star obviously implies an enhanced interaction rate and consequently higher luminosity of IC gamma rays $\left(L_{\gamma} \propto L_{*}\right)$. The Fermi and AGILE gammaray missions should be able to detect, at epochs close to the periastron, the line-type emission of the unshocked pulsar wind (Kirk et al. 1999; Ball \& Kirk 2000; Khangulyan et al. 2007), and in this way measure its Lorentz factor and mechanical power.

At first glance, the enhanced luminosity of the optical star should have a weaker impact on the gamma-ray emission 
related to the termination of the pulsar wind, since gammaray production is expected to proceed in the saturation regime. However, the enhanced optical luminosity does affect the gamma-ray emission for two reasons. First, it introduces a significant orbital dependence of the gamma-ray flux due to the Compton deceleration of the unshocked pulsar wind. This effect has been realized by Khangulyan et al. (2007), who indicated that the deficit of VHE emission observed by HESS close to the periastron passage may be explained by this effect, provided that the luminosity of the optical star is as large as $\sim 4 \times 10^{38} \mathrm{erg} \mathrm{s}^{-1}$. Although the value found here is smaller by a factor 1.7 , the Compton drag effect should be combined with gamma-gamma absorption, since for the updated orbital inclination the maximal attenuation (by a factor of two) occurs close to periastron passage. We note, however, that given the large orbital separation the total absorbed energy will be small, thus the contribution from the electromagnetic cascade should be negligible.

Finally, the production rate of VHE gamma rays is affected by the increase of the stellar temperature and the change of the orbital inclination. The higher photon temperature leads to a more pronounced impact of the Klein-Nishina effect. This, together with the smaller orbital inclination obtained here, should result in a weaker orbital phase dependence of the VHE gamma-ray production, in agreement with recent HESS observations (Aharonian et al. 2009; Kerschhaggl 2011).

It is clear that the new physical parameters of LS 2883 reported here have to be taken into account in the interpretation of the high- and very high-energy gamma-ray observations of the gamma-ray binary LS 2883/PSR B1259-63, such as the multiwavelength campaigns close to the 2010 December periastron passage, which include for the first time the participation of the Fermi and AGILE gamma-ray missions.

We thank Dr. I. D. H. Howarth and the referee, Dr. J. Puls, for very valuable suggestions, and J. Moldón for useful comments. This research is partially supported by the Spanish MICINN (grants FPA2010-22056-C06-02, AYA2008-06166-C03-01/03, AYA2010-21697-C05-04/05, and CSD2006-70 and FEDER funds); and by the Generalitat Valenciana (ACOMP/2009/164) and Gobierno de Canarias (ProID2010119). M.R. acknowledges financial support from MICINN and European Social Funds through a Ramón y Cajal fellowship.

Facility: VLT:Kueyen(UVES)

\section{REFERENCES}

Aharonian, F., et al. 2005, A\&A, 442, 1

Aharonian, F., et al. 2009, A\&A, 507, 389
Ball, L., \& Kirk, J. G. 2000, Astropart. Phys., 12, 335

Brand, J., \& Blitz, L. 1993, A\&A, 275, 67

Collins, G. W., Truax, R. J., \& Cranmer, S. R. 1991, ApJS, 77, 541

Cranmer, S. R., \& Owocki, S. P. 1995, ApJ, 440, 308

Dachs, J., Engels, D., \& Kiehling, R. 1988, A\&A, 194, 167

Dekker, H., D’Odorico, S., Kaufer, A., Delabre, B., \& Kotzlowski, H. 2000, Proc. SPIE, 4008, 534

Drilling, J. S. 1991, ApJS, 76, 1033

Dubus, G. 2006, A\&A, 456, 801

Fitzgerald, M. P. 1970, A\&A, 4, 234

Fremat, Y., Zorec, J., Hubert, A.-M., \& Floquet, M. 2005, A\&A, 440, 305

Grove, J. E., Tavani, M., Purcell, W. R., Johnson, W. N., Kurfess, J. D., Strickman, M. S., \& Arons, J. 1995, ApJ, 447, L113

Herbig, G. H. 1993, ApJ, 407, 142

Humphreys, R. M. 1978, ApJS, 38, 309

Hunter, I., Smoker, J. V., Keenan, F. P., Ledoux, C., Jehin, E., Cabanac, R., Melo, C., \& Bagnulo, S. 2006, MNRAS, 367, 1478

Johnston, S., Ball, L., Wang, N., \& Manchester, R. N. 2005, MNRAS, 358, 1069

Johnston, S., Manchester, R. N., Lyne, A. G., Bailes, M., Kaspi, V. M., Qiao, G., \& D'Amico, N. 1992, ApJ, 387, L37

Johnston, S., Manchester, R. N., Lyne, A. G., Nicastro, L., \& Spyromilio, J. 1994, MNRAS, 268, 430

Kaltcheva, N. T., \& Georgiev, L. N. 1994, MNRAS, 269, 289

Kaper, L., van der Meer, A., \& Najarro, F. 2006, A\&A, 457, 595

Kerschhaggl, M. 2011, A\&A, 525, A80

Khangulyan, D., Hnatic, S., Aharonian, F., \& Bogovalov, S. 2007, MNRAS, 380,320

Kirk, J. G., Ball, L., \& Skjaeraasen, O. 1999, Astropart. Phys., 10, 31

Klare, G., \& Neckel, T. 1977, A\&AS, 27, 215

Martins, F., Schaerer, D., \& Hillier, J. 2005, A\&A, 436, 1049

McClure-Griffiths, N. M., Dickey, J. M., Gaensler, B. M., \& Green, A. J. 2002, ApJ, 578, 176

Melatos, A., Johnston, S., \& Melrose, D. B. 1995, MNRAS, 275, 381

Moldón, J., Johnston, S., Ribó, M., Paredes, J. M., \& Deller, A. 2011, ApJ, 732, L10

Nyman, L.-A., Bronfman, L., \& Thaddeus, P. 1989, A\&A, 216, 185

Puls, J., Urbaneja, M. A., Venero, R., Repolust, T., Springmann, U., Jokuthy, A., \& Mokiem, M. R. 2005, A\&A, 435, 669

Rawlings, M. G., Adamson, A. J., \& Whittet, D. C. B. 2003, MNRAS, 341, 1121

Reig, P., Larionov, V., Negueruela, I., Arkharov, A. A., \& Kudryavtseva, N. A. 2007, A\&A, 462, 1081

Repolust, T., Puls, J., \& Herrero, A. 2004, A\&A, 415, 349

Ribó, M. 2008, in AIP Conf. Ser. 1010, A Population Explosion: The Nature and Evolution of X-ray Binaries in Diverse Environments, ed. R. M. Bandyopadhyay et al. (Melville, NY: AIP), 57

Schild, R. E., Garrison, R. F., \& Hiltner, W. A. 1983, ApJS, 51, 321

Simón-Díaz, S. 2010, A\&A, 510, A22

Stephenson, C. B., \& Sanduleak, N. 1971, Publ. Warner \& Swasey Obs., 1, 1

Stephenson, C. B., \& Sanduleak, N. 1977, ApJS, 33, 459

Tavani, M., et al. 1996, A\&AS, 120, 221

Townsend, R. H. D., Owocki, S. R., \& Howarth, I. D. 2004, MNRAS, 350, 189

Turner, D. G. 1980, ApJ, 240, 137

Uchiyama, Y., Tanaka, T., Takahashi, T., Mori, K., \& Nakazawa, K. 2009, ApJ, 698,911

Vink, J. S., de Koter, A., \& Lamers, H. J. G. L. M. 2000, A\&A, 362, 295

Westerlund, B. E., \& Garnier, R. 1989, A\&AS, 78, 203

Winkler, H. 1997, MNRAS, 287, 481 